\title{
The effect of horizontal and vertical furcation involvement on molar survival: a retrospective study
}

\section{Running title: Survival of furcation-involved molars}

\author{
Nibali L ${ }^{1}$, Sun $\mathrm{C}^{2}$, Akcalı $\mathrm{A}^{1}$, Yeh $\mathrm{YC}^{3}$, Tu YK ${ }^{3}$, Donos $\mathrm{N}^{1}$ \\ ${ }^{1}$ Centre for Immunobiology \& Regenerative Medicine and Centre for Oral Clinical Research, \\ Institute of Dentistry, Barts and the London School of Medicine and Dentistry, Queen Mary \\ University of London (QMUL), London, United Kingdom \\ ${ }^{2}$ Department of Periodontology, Faculty of Dentistry, Suzhou Health College, Suzhou, China \\ ${ }^{3}$ Institute of Epidemiology and Preventive Medicine, College of Public Health, National \\ Taiwan University, Taipei, Taiwan
}

\# Corresponding author:

Luigi Nibali

Centre for Oral Clinical Research, Institute of Dentistry

Queen Mary University of London (QMUL)

London, United Kingdom

Tel.+44 207882 3134, 1.nibali@qmul.ac.uk

Number of figures: 1

Number of tables: 6

Abstract word count: 181

Total word count: 3328

KEYWORDS: Periodontitis, progression, maintenance, stability, furcation

FUNDING: No specific funding was obtained for this study. 
CONFLICTS OF INTEREST: The authors have stated explicitly that there are no conflicts of interest in connection with this article.

\begin{abstract}
Background: Periodontal involvement of the furcation area (furcation involvement, FI) has been reported to confer to molars a higher risk of tooth loss.

Aims: The aim of this retrospective analysis was to assess the effect of FI on disease progression and tooth loss in molars of patients with chronic periodontitis undergoing supportive periodontal therapy (SPT) in a UK private practice setting.

Materials and Methods: Six-hundred and thirty-three molars were analysed in 100 chronic periodontitis patients treated with active periodontal therapy (APT) and followed up in SPT for at least 5 years. Molars were treated with a combination of resective, regenerative or conservative approaches, according to the different clinical needs.

Results: Twenty-three molars were extracted during APT and a further 23 were lost during SPT. Multivariable analysis showed that both horizontal FI and vertical furcation component were associated with increased risk of tooth loss during SPT (OR 5.26, 95\% CI 1.46-19.03, p=0.012 and OR 9.83, 95\% CI 1.83-50.11, p=0.006 respectively).
\end{abstract}

Conclusion: Attention should be placed on both horizontal and vertical furcation involvement in molars, owing to their association with tooth loss during SPT.

\title{
CLINICAL RELEVANCE
}

Scientific rationale for the study: Furcation involvement has consistently been associated with tooth loss.

Principal findings: Both horizontal and vertical furcation involvement are associated with molar loss in patients with chronic periodontitis under regular SPT.

Practical implications: Attention should be paid to the prevention and treatment of both horizontal and vertical furcation involvement in order to reduce risk of molar loss. 


\section{INTRODUCTION}

Periodontal furcation involvement (FI) represents a challenge in the treatment of periodontally-compromised molars. Different treatment modalities are advocated for the treatment of FI, including non-surgical maintenance, resective and regenerative treatment, according to subject and site factors and degree of involvement of individual affected molars. A systematic review on long-term tooth loss related to FI revealed that the survival rate of molars treated non-surgically was $>90 \%$ after $5-9$ years, with different breakdowns according to treatment protocols and varying degrees of disease severity and that vertical root fractures and endodontic failures were the most frequent complications observed following resective procedures (Huynh-Ba et al. 2009). A more recent systematic review and meta-analysis of studies including periodontal treatment and at least 3 years of supportive periodontal therapy (SPT) concluded that the average tooth loss/year was 0.01 and 0.02 respectively for molars without and with horizontal FI (Nibali et al. 2016). Based on this metaanalysis, FI approximately doubles the risk of tooth loss for molars in SPT for up to 10-15 years. In particular, molars with FI had a relative risk (RR) of tooth loss of 1.46 $(p=0.06)$ up to 10 years and of 2.21 from 10 to 15 years $(p<0.0001)$ compared with molars with no FI. Sparse data are available on the potential effect of different degrees of vertical FI on tooth loss risk (Tonetti et al. 2017).

The aim of the present study was to assess the outcome of treatment and the survival of molars with FI in a cohort of patients with chronic periodontitis following periodontal treatment and maintenance care in a private practice setting in the UK.

\section{MATERIALS AND METHODS}

\section{Patient population}

The patient population has been described elsewhere (Nibali et al. 2017). Briefly, 100 consecutive patients with at least 5 years of maintenance care were selected from author LN's patient list in 3 private periodontal practices in London and Bishop's Stortford, United Kingdom. Ethics approval for the analysis was sought from The LondonandCity Ethics Committee, which gave permission for the study to be carried out as service evaluation (reference 14 LO 0629). All patients had been diagnosed 
with chronic periodontitis (Lindhe et al. 1999), ranging from localised to generalised, with interproximal attachment loss $\geq 3 \mathrm{~mm}$ in at least 2 non-adjacent teeth (Tonetti \& Claffey 2005) and at least 2 sites with $\geq 5 \mathrm{~mm}$ probing pocket depths (PPDs) and radiographic evidence of bone loss $\geq 20 \%$ of root length at first visit. Clinical characteristics have been previously described (Nibali et al. 2017). All patients were treated by author LN with non-surgical periodontal treatment with or without subsequent periodontal surgeries and then reassessed at least 5 years after completion of initial therapy (following at least 1/year SPT sessions).

\section{Clinical examinations}

Clinical and radiographic data from all patients and all patient visits were obtained and analysed as described before (Nibali et al. 2017). Dichotomous full mouth plaque scores (FMPS) (Guerrero et al. 2005), full mouth probing pocket depth (PPD), recession (REC) of the gingival margin from the cemento-enamel junction (CEJ), bleeding on probing (FMBS) (Guerrero et al. 2005), tooth mobility (Laster et al. 1975) and horizontal furcation involvement (Hamp et al. 1975) were measured at baseline, end of active periodontal therapy (APT) and at the last study visit. Clinical attachment level (CAL) was calculated as PPD+REC. Clinical parameters were assessed by gentle probing using a UNC-15 periodontal probe and a Nabers probe for furcations. Dental radiographs of each patient had been obtained as necessary for diagnosis and treatment planning purposes at this visit.

APT consisted of oral hygiene instructions and non-surgical supra- and sub-gingival debridement followed by, when indicated, additional periodontal surgery and conservative, endodontic and prosthetic treatment if necessary. According to the initial treatment plan, teeth which were considered hopeless were extracted during APT. The definition of 'hopeless' has been extensively discussed previously (Nibali et al. 2017) and was generally based on bone loss $\geq 70 \%$, with degree III FI or grade III mobility, or unrestorable. A total of 9 patients received systemic antibiotics during APT (non-surgical or surgical phase). Following APT, patients were entered in SPT, consisting of visits at an individualized interval of 3-12 months for medical and dental history updates, clinical and (if considered necessary) radiographic data collection, 
oral hygiene re-instructions and motivation and supra- and sub-gingival debridement (under local anaesthesia when necessary). The frequency of SPT visit was tailored to patient needs as judged by the treating clinician (author LN). Additional visits with the hygienists were arranged for some of the patients. If deterioration in periodontal parameters was detected, further treatment (including periodontal surgeries, extractions or endodontic therapy) was carried out.

\section{Reproducibility of furcation measurement}

A total of 98 molar furcations in 6 patients were measured twice with a Nabers probe with at least 15 minutes' separation by the study examiner (LN). The FI scores ranged from no FI to degree III FI, with a Kappa score of 0.96 , indicating very good reproducibility.

\section{Radiographic analyses}

Periapical radiographs from all patients included in the study were screened, entered in a dedicated database, transferred into a dedicated software system (Xposeit version 3.01, Torben Jørgensen, Lystrup, Denmark) and analysed by one designated examiner (author AA) as described before (Nibali et al. 2017). In addition to that, vertical furcation involvement was classified based on percentage of bone loss (BL) in the furcation area, as subclass A (BL extending to the coronal third or $\leq 33 \%$ ), B (BL extending to the middle third or $>33 \%-67 \%$ BL) or C (BL extending to the apical third or $\geq 67 \%$ BL) (Tarnow and Fletcher 1984). For maxillary molars, the value relative to the worst affected root, as estimated in periapical radiographs, was used. The presence of intrabony defects on the studied molars was detected radiographically as described before (Nibali et al. 2011). Reproducibility of the radiographic assessments was performed by obtaining 2 separate measurements of root length and bone defect depth radiographic parameters on 50 radiographs 1-week apart. The intraclass correlation for defect depth was 0.99 , showing very good reproducibility.

\section{Furcation treatment}

All molars with FI were initially treated by non-surgical therapy, using ultrasonic and hand instruments. After initial re-evaluation, according to degree of FI, patient and 
site characteristics as well as strategic considerations, some molars were considered as good candidates for surgical intervention. Regenerative surgery (with either guided tissue regeneration, GTR, or enamel matrix derivatives, EMD) was generally performed in cases of degree II FI with good interproximal bone levels, reduced vertical furcation component and narrow furcation width (Pontoriero et al. 1992, Bowers et al. 2003, Horwitz et al. 2004), as well as in the presence of intrabony defects. Root resection surgery was only applied in one case of single degree III FI, in order to eliminate the furcation lesion. OFD was performed in other cases where it was thought that resective or regenerative options were not appropriate or not agreed on by the patient, but it was felt that surgical access to the furcation lesion could improve debridement. In cases where oral hygiene dexterity and motivation were good and the root anatomy appeared favourable (short root trunk and high root divergence) (Muller \& Eger 1999), tunnelling surgery was preferred.

\section{Statistical analysis}

Data from all patients were entered into a spreadsheet and proofed for entry errors. Continuous variables are reported as means and standard deviations. Two outcomes were evaluated. The primary outcome was the incidence of tooth loss during SPT (for teeth with different degrees of FI). The secondary outcomes were changes in periodontal clinical parameters including average CAL, rate of bone loss in mesial or distal sites during APT and SPT. Multilevel models were used in order to take into account the correlation among repeated measures within the same teeth and teeth within the same patient. All analyses used two-sided tests, and the level of statistical significance was set at $5 \%$. Univariate analyses were initially performed. Due to concern of collinearity between CAL, bone loss and both horizontal and vertical furcation involvement (and also between endodontic therapy and presence of restorations), not all parameters were included in the final multivariable model. All our statistical analysis was performed using SAS, version 9.4.

\section{RESULTS}

\section{Baseline characteristics}


The demographic and clinical characteristics of all patients included in the study have been reported previously (Nibali et al. 2017). The great majority of patients were Caucasians (93\%), prevalently female and with an average age of approximately 53 years. Twenty-two per cent of patients were former smokers and $20 \%$ current smokers at baseline (average 12 cigarettes/day for 29 years). A total of 30 patients reported a diagnosis of a medical conditions, the most common being hypertension (13\%). The total observation period during SPT was 79.1 \pm 18.0 months (range 60-123 months).

A total of 633 molars (excluding third molars) were included in the study. Table 1 shows clinical and radiographic characteristics of included molars at baseline. Overall, 283 molars (45\%) had FI (Hamp et al. 1975) diagnosed at baseline, with decreasing numbers from degree I $(n=156)$ to degree II $(n=79)$ to III $(n=48)$. The majority of molars had bone loss in the coronal third of the furcation area (subclass A, $57.5 \%$ ), with $33.5 \%$ subclass $\mathrm{B}$ and $9 \%$ subclass $\mathrm{C}$ (bone loss to the apical third). A total of 98 molars $(24.5 \%)$ also had intrabony defects affecting at least one root. Table 2 shows FI divided by tooth type, highlighting more severe furcation involvement for upper vs. lower molars.

The treatment breakdown according to furcation diagnosis is presented in figure 1 . The great majority of molars with no FI and with degree I FI did not undergo surgery. OFD or regenerative surgery or extractions were occasionally performed for reasons other than furcation involvement (e.g. persisting PPD or intrabony defect). Equally, some extractions were carried out for molars with no FI or degree I FI (e.g. for endodontic pathology). Eighteen per cent and 5\% of degree II furcation molars underwent OFD and tunnelling respectively, while none of them were treated with regenerative surgery. Among degree III FI molars, 13 were extracted, 12 underwent tunnelling surgery, 1 underwent root resection and the rest were treated nonsurgically. A total of 12 molars ( 6 of which with FI degree I-II) received regenerative periodontal surgery, with either GTR or EMD.

Tables 3 reports the association between clinical and radiographic characteristics and furcation involvements at baseline. Shorter root length was associated with worse 
furcation involvement $(\mathrm{p}<0.001)$. As expected, FI was also associated with higher PPD, CAL, mobility, bone loss and vertical furcation involvement (all $\mathrm{p}<0.001$ ). No statistically significant association was detected at baseline between endodontic status or presence of intrabony defects and FI.

\section{Changes in furcation diagnosis and tooth loss during APT and SPT}

During APT, 23 molars were extracted. The majority of molars with no FI were still diagnosed with no FI after APT (see table 4). The majority of molars with FI I at baseline converted to no FI, while most others remained as FI I. Among FI II molars at baseline, there was an equal number of degree I and degree II at the end of APT, with $26 \%$ going down to no FI at end of SPT and 3 cases converting from FI II to FI III. All molars with degree III FI at baseline were still diagnosed with degree III at end of APT, with the exception of one which underwent root resection.

During SPT, 23 molars were extracted. The breakdown of extraction by FI was $1.4 \%$, $7.9 \%, 12.8 \%$ and $8.1 \%$ respectively for molars with no FI, degree I, degree II and degree III FI. The majority of molars (approximately 65\%) maintained their FI diagnosis throughout SPT, while $23 \%$ worsened and $8 \%$ improved in the furcation degree, in addition to the 23 molars (4\%) which were extracted. Of the 23 molars extracted during SPT, 19 had only received subgingival debridement as part of APT and SPT, 3 had undergone OFD and 1 had undergone regenerative surgery.

\section{Effect of FI on tooth loss during SPT}

Table 5 shows associations between patient- and tooth-based parameters and molar loss. In the univariate model, FI at start of SPT, previous endodontic therapy, vertical furcation component, average CAL and average bone loss were al associated with molar loss during SPT. In the multivariable analysis, FI was associated with molar loss (OR 5.26, 95\% CI 1.46-19.03, p=0.012). Also, vertical furcation component was associated with molar loss (OR 9.83, 95\% CI 1.83-50.11, p=0.006).

Both degree I FI and degree II-III FI were associated with tooth loss during SPT compared with no FI at start of SPT (OR 6.49, 95\% CI 2.17-19.37 and OR 7.26, 95\% 
CI 2.24-23.51, both $\mathrm{p}<0.001)$. In the multivariable model, the association for degree I FI was statistically significant (OR 7.02, 95\% CI 1.63-30.26, p=0.009), while for degree II-III FI statistical significance was not reached (OR 3.95, 95\% CI 0.88-17.73, $\mathrm{p}=0.072$ ). A gradient effect was observed for vertical furcation component and molar loss (OR 6.72, 95\% CI 1.18-38.22, p=0.032 and OR 34.94, 95\% CI 4.52-270.00, $\mathrm{p}<0.001$ respectively for subclasses B and C vs. A).

When using the baseline parameters (rather than parameters at start of SPT) (see supplementary tables 1 and 2), FI, previous endodontic therapy, vertical furcation component, average CAL and average bone loss were all associated with molar loss during SPT.

To evaluate the association of baseline furcation involvement and changes in average CAL, and rates of bone loss at mesial or distal sites during APT and SPT, the different linear slopes for furcation involvements in different times were evaluated. Table 6 reports results of multilevel model for association with baseline FI and changes in CAL, \% mesial bone loss and \% distal bone loss. The interaction model showed that the estimated average changes of CAL at APT and SPT were not significantly different between degree I FI and no FI. The estimated changes of CAL at APT and SPT were $-0.64 \mathrm{~mm}(95 \% \mathrm{CI}:-1.03,-0.24)$ and $-0.63 \mathrm{~mm}(-1.09,-0.17)$ in favour of molars with degree II FI compared with no FI. The estimated change of CAL at APT and SPT for molars with degree III FI were $-1.41 \mathrm{~mm}$ (95\%CI: -1.94, -0.89) and -0.93 mm (-1.55, -0.31 ) in favour of molars with degree III FI compared with no FI.

\section{DISCUSSION}

The analysis reported here aimed to investigate the destiny of maxillary and mandibular molars with and without furcation involvement during an average 7 years (minimum 5) of supportive periodontal therapy. The results agree with previous literature in confirming a higher risk of tooth loss for molars affected by furcation involvement at the start of SPT. This risk was estimated to be 2-4 times higher than molars with no FI in a recent systematic review (Nibali et al. 2016). In the present 
study, molars with FI were around 5 times more likely to be extracted during SPT than molars with no FI in the multivariable analysis. This risk was higher when vertical furcation component was considered.

The sample described here comes from a study showing an average tooth loss of 0.06 teeth/patient/year in a cohort undergoing strict periodontal maintenance care. It is important to highlight that out of 23 molars lost during SPT, only 8 were lost for periodontal reasons ( 8 for endodontic reasons, 6 for fractures and 1 for caries). Therefore, although both horizontal and vertical furcation involvement are associated with molar loss, other factors such as previous endodontic therapy are also very important. Furthermore, bone loss and average CAL were associated with molar loss. Owing to collinearity, not all these factors were added to the final multivariable model, which makes it difficult to ascertain the relative contribution of each individual variable on molar loss risk.

Most previous studies investigating the different effects of degree of FI on tooth loss had concluded that a gradient effect exists increasing from degree I to II and then to III (McGuire \& Nunn 1996, Dannewitz et al. 2006, Johansson et al. 2013, Salvi et al. 2014, Graetz et al. 2015). A meta-analysis of this association concluded that relative risk of tooth loss was approximately 1.67 for degree II vs. I, 1.83 for III vs. II and 3.13 for degree III vs. I (Nibali et al. 2017). In the present study, the breakdown of extraction by FI was $1.4 \%, 7.9 \%, 12.8 \%$ and $8.1 \%$ respectively for molars with no FI, degree I, degree II and degree III FI during SPT. A statistical significant association was found only for degree I FI vs. no FI. It is likely that the absence of statistical significance for degree II and III may be due to the relatively small number of teeth with this degree of FI. The discrepancy between this and previous studies might also be due to the treatment rendered as part of this study and perhaps to a higher attention given to try and maintain degree III FI molars.

Furcation diagnosis in the current study was performed by using curved Nabers probes with the aid of periapical radiographs. The accuracy of both methods is not ideal (Eickholz \& Kim 1998, Walter et al. 2012), especially in deeper furcations, and the reliability of clinical assessment depends on the anatomy and location of the tooth 
(Graetz et al. 2014). However, the combined use of clinical and radiographic methods is probably the most practical way for furcation diagnosis (Graetz et al. 2014), since intra-surgical measurements and three-dimensional radiographs can only be used in specific cases (Walter et al. 2012). The accuracy of the clinical furcation measurements in this study was confirmed by the examiner's reproducibility exercise. It has been shown that examiner's experience has a strong impact on reliability of radiographic FI diagnosis (Graetz et al. 2014). The degree of FI detected clinically and radiographically formed the basis for treatment planning and yielded favourable long-term survival rates. This stresses the importance of special attention to be given to furcation diagnosis and treatment planning, including non-surgical and surgical approaches according to treatment needs and strict adherence to supportive periodontal therapy.

It is interesting to notice that vertical furcation component calculated on radiographs (Tarnow and Fletcher 1984) was also associated with molar loss during SPT. This is in agreement with a recent study showing a gradually higher incidence of tooth loss at 10 years for degree II FI with bone loss up to the apical third, middle third or coronal third of the root (Tonetti et al. 2017). The authors advocated that the vertical component might be an important predictor of tooth loss in molars with degree II horizontal furcation involvement (Tonetti et al. 2017).

At baseline, an association was detected between shorter root length and worse furcation involvement $(\mathrm{p}<0.001)$, which confirms the notion that FI is more likely in molars where the entrance to the furcation area is closer to gingival margin and hence to the microbial front. As expected, baseline FI was also associated with higher PPD, CAL, mobility, bone loss and vertical furcation involvement, whilst no statistically significant association was detected at baseline between endodontic status or presence of intrabony defects and FI.

The strengths of this study lie in the homogeneity of having one single therapist and examiner in a private practice setting, with precise data on reproducibility and on reasons for extraction, in the use of a multilevel analysis to take into account patient- 
and tooth-factors and in the analysis of both horizontal and vertical furcation involvement. The main limitations are the retrospective nature of the study, with the consequent potential bias, the relatively small sample size and the reduced external validity and potential bias due to one single clinician being both therapist and examiner. The average nearly 7 years follow-up may not be long enough to draw definitive conclusions on tooth survival, as the risk of tooth loss in FI-involved molars seems to increase with time (Nibali et al. 2016). Furthermore, determination of radiographic vertical furcation involvement in 3-roted maxillary molars is perhaps less reliable than in mandibular molars. In conclusion, this study confirms that both horizontal and vertical furcation involvement are associated with tooth loss and should be considered as factors in personalised maintenance programs (Lang et al. 2015).

\section{REFERENCES}

- Bowers, G.M., Schallhorn, R.G., McClain, P.K., Morrison, G.M., Morgan, R. \& Reynolds, M.A. (2003) Factors influencing the outcome of regenerative therapy in mandibular class II furcations: Part I. Journal of Periodontology 74, 1255-1268.

-Dannewitz, B., Zeidler, A., Hüsing, J., Saure, D., Pfefferle, T., Eickholz, P. \& Pretzl, B. (2016) Loss of molars in periodontally treated patients: results 10 years and more after active periodontal therapy. Journal of Clinical Periodontology 43, 53-62.

- Eickholz, P. \& Kim, T.-S. (1998) Reproducibility and validity of the assessment of clinical furcation parameters as related to different probes. Journal of Periodontology 69, 328-336.

- Graetz, C., Schutzhold, S., Plaumann, A., Kahl, M., Springer, C., Salzer, S., Holtfreter, B., Kocher, T., Dorfer, C. E. \& Schwendicke, F. (2015) Prognostic factors for the loss of molars - an 18-years retrospective cohort study. Journal of Clinical Periodontology 42, 943-950..

- Guerrero, A., Griffiths, G. S., Nibali, L., Suvan, J., Moles, D. R., Laurell, L. \& Tonetti, M. S. (2005) Adjunctive benefits of systemic amoxicillin and metronidazole in non-surgical treatment of generalized aggressive periodontitis: a randomized placebo-controlled clinical trial. Journal of Clinical Periodontology 32: 1096-1107.

- Hamp, S., Nyman, S. \& Lindhe, J. (1975) Periodontal treatment of multirooted teeth. Results after 5 years. Journal of Clinical Periodontology 2: 126-135. 
- Horwitz, J., Machtei, E.E., Reitmeir, P., Holle, R., Kim, T.S., Eickholz, P. (2004) Radiographic parameters as prognostic indicators for healing of class II furcation defects. Journal of Clinical Periodontology 31, 105-111.

- Huynh-Ba, G., Kuonen, P., Hofer, D., Schmid, J., Lang, N. P. \& Salvi, G. E. (2009) The effect of periodontal therapy on the survival rate and incidence of complications of multirooted teeth with furcation involvement after an observation period of at least 5 years: a systematic review. Journal of Clinical Periodontology 36, 164-176.

- Johansson, K. J., Johansson, C. S. \& Ravald, N. (2013) The prevalence and alterations of furcation involvements 13 to 16 years after periodontal treatment. Swedish Dental Journal 37, 87-95.

- Lang, N.P., Suvan, J.E., \& Tonetti, M.S. (2015) Risk factor assessment tools for the prevention of periodontitis progression a systematic review. Journal of Clinical Periodontology 42: 59-70

- Laster, L., Laudenbach, K. \& Stoller, N. (1975) An evaluation of clinical tooth mobility measurements. Journal of Periodontology 46: 603-607.

- Lindhe, J., Ranney, R., Lamster, R.I., Charles, A., Chung, C., Flemming, T. Kinane, D., Listgarten, M., Löe, H., Schoor, R., Seymour, G. \& Somerman, M. (1999) Consensus report: Chronic periodontitis. Annals of Periodontology 4: 38.

- McGuire, M.K. \& Nunn, M.E. (1996) Prognosis versus actual outcome. III. The effectiveness of clinical parameters in accurately predicting tooth survival. Journal of Periodontology 67: 666-674.

- Muller, H. P. \& Eger, T. (1999) Furcation diagnosis. Journal of Clinical Periodontology 26, 485-498.

- Nibali, L., Pometti, D., Tu, Y.K., \& Donos, N. (2011) Clinical and radiographic outcomes following non-surgical therapy of periodontal infrabony defects: a retrospective study. Journal of Clinical Periodontology 38: $50-57$.

- Nibali, L., Zavattini, A., Nagata, K., Di Iorio, A., Lin, G.H., Needleman, I., \& Donos, N. (2016) Tooth loss in molars with and without furcation involvement - a systematic review and meta-analysis. Journal of Clinical Periodontology 43: 156-66.

- Nibali, L., Sun, C., Akcal1, A., Meng, X., Tu, Y.K. \& Donos, N. (2017) A retrospective study on periodontal disease progression in private practice. Journal of Clinical Periodontology 44, 290-297.

- Pontoriero, R., Nyman, S., Ericsson, I. \& Lindhe, J. (1992) Guided tissue regeneration in surgically-produced furcation defects. An experimental study in the beagle dog. Journal of Clinical Periodontology 19:159-63.

-Salvi, G. E., Mischler, D. C., Schmidlin, K., Matuliene, G., Pjetursson, B. E., Bragger, U. \& Lang, N. P. (2014) Risk factors associated with the longevity of multi-rooted teeth. Long-term outcomes after active and supportive periodontal therapy. Journal of Clinical Periodontology 41, 701-707.

- Tarnow, D. \& Fletcher, P. (1984) Classification of the vertical component of furcation involvement. Journal of Periodontology 55, 283-284.

- Tonetti, M., Christianes, A. \& Cortellini, P. (2017) Vertical sub-classification predicts survival of molars with class II Furcation involvement during 
supportive periodontal care. Journal of Clinical Periodontology Aug 3. doi: 10.1111/jcpe.12789. [Epub ahead of print].

- Tonetti, M.S. \& Claffey, N. (2005) Advances in the progression of periodontitis and proposal of definitions of a periodontitis case and disease progression for use in risk factor research. Group C consensus report of the 5th European Workshop in Periodontology. Journal of Clinical Periodontology 32: 210-213.

- Walter, C., Weiger, R., Dietrich, T., Lang, N. P. \& Zitzmann, N. U. (2012) Does three-dimensional imaging offers a financial benefit for the treatment of maxillary molars with furcation involvement? - a pilot clinical case series. Clinical Oral Implants Research 23, 351-358. 
Table 1. Clinical and radiographic characteristics of included molars at baseline

\begin{tabular}{|c|c|c|c|}
\hline Variable & & Number (\%) & Average \\
\hline $\begin{array}{l}\text { Number of first and } \\
\text { second molars }\end{array}$ & & $633(100.0)$ & \\
\hline \multirow[t]{2}{*}{ Average PPD } & Mean (SD) & 633 & $5.59(2.05)$ \\
\hline & Median (min, max) & & $5.00(2.00,15.00)$ \\
\hline \multirow[t]{2}{*}{ Average CAL } & Mean (SD) & 633 & $6.34(2.39)$ \\
\hline & Median (min, max) & & $6.00(2.00,16.00)$ \\
\hline \multirow[t]{4}{*}{ Mobility } & None & $547(86.4)$ & - \\
\hline & Degree I & $61(9.6)$ & - \\
\hline & Degree II & $14(2.2)$ & - \\
\hline & Degree III & $11(1.7)$ & - \\
\hline \multirow[t]{4}{*}{ Horizontal FI } & None & $345(54.5)$ & - \\
\hline & Degree I & $161(25.4)$ & - \\
\hline & Degree II & $79(12.5)$ & - \\
\hline & Degree III & $48(7.6)$ & - \\
\hline \multirow[t]{3}{*}{ Vertical FI } & A & $335(57.5)$ & - \\
\hline & $\mathrm{B}$ & $195(33.5)$ & - \\
\hline & $\mathrm{C}$ & $53(9.0)$ & - \\
\hline \multirow[t]{2}{*}{ Intrabony defect } & No & $302(75.5)$ & - \\
\hline & Yes & $98(24.5)$ & - \\
\hline \multirow[t]{2}{*}{$\%$ bone loss } & Mean (SD) & 424 & $0.32(0.15)$ \\
\hline & Median (min, $\max$ ) & & $0.28(0.03,1.12)$ \\
\hline
\end{tabular}


Table 2. Distribution of FI by molar type at baseline $(n=633)$

\begin{tabular}{|c|c|c|c|c|c|c|c|c|}
\hline \multirow[b]{3}{*}{ Molar type } & \multicolumn{8}{|c|}{ Furcation involvement } \\
\hline & \multicolumn{2}{|c|}{ None } & \multicolumn{2}{|c|}{ Degree I } & \multicolumn{2}{|c|}{ Degree II } & \multicolumn{2}{|c|}{ Degree III } \\
\hline & $n$ & $(\%)$ & $n$ & $(\%)$ & $n$ & $(\%)$ & $n$ & $(\%)$ \\
\hline $\begin{array}{l}\text { Maxillary first molar } \\
(\mathrm{n}=162)\end{array}$ & 75 & $(46.3)$ & 44 & $(27.2)$ & 21 & $(13.0)$ & 22 & $(13.6)$ \\
\hline $\begin{array}{l}\text { Maxillary second molar } \\
(\mathrm{n}=153)\end{array}$ & 66 & $(43.1)$ & 48 & $(31.4)$ & 22 & $(14.4)$ & 17 & $(11.1)$ \\
\hline $\begin{array}{l}\text { Mandibular first molar } \\
(\mathrm{n}=157)\end{array}$ & 94 & $(59.9)$ & 35 & $(22.3)$ & 20 & $(12.7)$ & 8 & $(5.1)$ \\
\hline $\begin{array}{l}\text { Mandibular second molar } \\
(\mathrm{n}=161)\end{array}$ & 110 & $(68.3)$ & 34 & (21.1) & 16 & $(9.9)$ & 1 & $(0.6)$ \\
\hline
\end{tabular}


Table 3. Associations of clinical and radiographic characteristics with furcation involvement at baseline. $\mathrm{P}$ values were calculated with Chi-square test or ANOVA for categorical and continuous variables respectively.

\begin{tabular}{|c|c|c|c|c|c|c|}
\hline \multirow[b]{3}{*}{ Variable } & \multicolumn{5}{|c|}{ Furcation involvement at baseline } & \multirow[b]{3}{*}{ Post hoc tests } \\
\hline & None & Degree I & Degree II & Degree III & & \\
\hline & $n(\%)$ & $n(\%)$ & $n(\%)$ & $n(\%)$ & $P$ value & \\
\hline \multicolumn{7}{|l|}{ Root length(mm) } \\
\hline Mean(SD) & $14.52(2.03)$ & $13.82(1.84)$ & 13.91(1.84) & $13.51(1.67)$ & 0.001 & None $>I$ \\
\hline Median (min,max) & $14.63(8.62,19.93)$ & $13.82(8.60,19.63)$ & $13.96(8.94,18.07)$ & $13.96(9.11,16.66)$ & & None>III \\
\hline \multicolumn{7}{|l|}{ Average PPD } \\
\hline $\operatorname{Mean}(\mathrm{SD})$ & $4.98(1.80)$ & $5.59(1.77)$ & $6.86(1.84)$ & $7.85(2.43)$ & $<0.001$ & All \\
\hline Median (min,max) & $5.00(2.00,12.00)$ & $6.00(2.00,12.00)$ & $7.00(3.00,11.00)$ & $7.00(5.00,15.00)$ & & \\
\hline \multicolumn{7}{|l|}{ Average CAL } \\
\hline $\operatorname{Mean}(\mathrm{SD})$ & $5.39(1.88)$ & $6.45(1.80)$ & $8.00(1.93)$ & $10.13(2.76)$ & $<0.001$ & All \\
\hline Median (min,max) & $5.00(2.00,14.00)$ & $6.00(3.00,12.00)$ & $8.00(3.00,12.00)$ & $10.00(5.00,16.00)$ & & \\
\hline \multicolumn{7}{|l|}{ Mobility } \\
\hline None & $325(94.20)$ & 144(89.44) & $57(72.15)$ & $21(43.75)$ & $<0.001$ & \\
\hline Degree I & $18(5.22)$ & $14(8.70)$ & $13(16.46)$ & $16(33.33)$ & & \\
\hline Degree II & $2(0.58)$ & $2(1.24)$ & $8(10.13)$ & $2(4.17)$ & & \\
\hline Degree III & & $1(0.62)$ & $1(1.27)$ & $9(18.75)$ & & \\
\hline \multicolumn{7}{|c|}{ Vertical furcation involvement } \\
\hline A & $232(72.5)$ & $74(51.4)$ & $24(32.4)$ & $5(11.1)$ & $<0.001$ & \\
\hline B & $77(24.1)$ & $58(40.3)$ & $38(51.4)$ & $22(48.9)$ & & \\
\hline $\mathrm{C}$ & $11(3.4)$ & $12(8.3)$ & $12(16.2)$ & $18(40.0)$ & & \\
\hline \multicolumn{7}{|l|}{ Intrabony defect } \\
\hline No & $161(78.2)$ & 78(78.0) & $40(67.8)$ & $23(65.7)$ & 0.185 & \\
\hline Yes & $45(21.8)$ & $22(22.0)$ & $19(32.2)$ & $12(34.3)$ & & \\
\hline \multicolumn{7}{|l|}{$\%$ bone loss } \\
\hline Mean(SD) & $0.26(0.11)$ & $0.32(0.13)$ & $0.38(0.15)$ & $0.53(0.20)$ & $<0.001$ & All \\
\hline Median (min,max) & $0.23(0.03,0.71)$ & $0.30(0.12,0.70)$ & $0.36(0.15,0.96)$ & $0.53(0.18,1.12)$ & & \\
\hline \multicolumn{7}{|l|}{ Endodontic therapy } \\
\hline No & 210(94.59) & $100(88.50)$ & $59(95.16)$ & $37(88.10)$ & 0.121 & \\
\hline Yes & $12(5.41)$ & $13(11.50)$ & $3(4.84)$ & $5(11.90)$ & & \\
\hline
\end{tabular}


Table 4. Change of furcation involvements during APT and SPT. P values were calculated with Chi-square test.

\begin{tabular}{|c|c|c|c|c|c|c|c|c|c|c|}
\hline \multirow[b]{3}{*}{ Variable } & \multicolumn{10}{|c|}{ Furcation involvement at baseline } \\
\hline & \multirow{2}{*}{$\begin{array}{c}\text { Total } \\
n\end{array}$} & \multicolumn{2}{|c|}{ None } & \multicolumn{2}{|c|}{ Degree I } & \multicolumn{2}{|c|}{ Degree II } & \multicolumn{2}{|c|}{ Degree III } & \multirow[b]{2}{*}{$P$ value } \\
\hline & & $n$ & $(\%)$ & $n$ & $(\%)$ & $n$ & $(\%)$ & $n$ & $(\%)$ & \\
\hline \multicolumn{11}{|c|}{$\begin{array}{l}\text { Furcation involvement at } \\
\text { Visit } 2\end{array}$} \\
\hline None & 414 & 309 & $(91.2)$ & 84 & $(53.8)$ & 20 & $(27.8)$ & & $(2.9)$ & $<0.001$ \\
\hline Degree I & 114 & 27 & $(8.0)$ & 62 & $(39.7)$ & 25 & $(34.7)$ & & & \\
\hline Degree II & 37 & 3 & $(0.9)$ & 10 & $(6.4)$ & 24 & $(33.3)$ & & & \\
\hline Degree III & 37 & & & & & 3 & $(4.2)$ & 34 & $(97.1)$ & \\
\hline \multicolumn{11}{|c|}{$\begin{array}{l}\text { Furcation involvement at } \\
\text { Visit } 3\end{array}$} \\
\hline None & 300 & 231 & $(72.9)$ & 54 & $(38.6)$ & 14 & (20.9) & 1 & $(3.3)$ & $<0.001$ \\
\hline Degree I & 168 & 79 & $(24.9)$ & 65 & (46.4) & 24 & $(35.8)$ & & & \\
\hline Degree II & 40 & 7 & $(2.2)$ & 17 & (12.1) & 16 & (23.9) & & & \\
\hline Degree III & 46 & & & & (2.9) & 13 & (19.4) & 29 & (96.7) & \\
\hline
\end{tabular}


Table 5. Odds ratio of tooth loss during SPT considering clinical parameters at start of SPT. The univariate model is presented in the first column and two multivariable models in the next two columns. Not all parameters are entered in the models because of collinearity.

\begin{tabular}{|c|c|c|c|c|c|c|}
\hline \multirow[b]{3}{*}{ Variable } & & & \multicolumn{4}{|c|}{ Multivariable models } \\
\hline & \multicolumn{2}{|c|}{ Univariate model } & \multicolumn{2}{|l|}{ Model 1} & \multicolumn{2}{|l|}{ Model 2} \\
\hline & $O R(95 \% C I)$ & $P$ value & $O R(95 \% C I)$ & $P$ value & OR $(95 \% C I)$ & $P$ value \\
\hline \multicolumn{7}{|l|}{ Gender } \\
\hline Female & Reference & & Reference & & Reference & \\
\hline Male & $1.23(0.47,3.19)$ & 0.669 & $1.28(0.35,4.63)$ & 0.704 & $1.83(0.53,6.25)$ & 0.334 \\
\hline Age & $1.05(0.99,1.11)$ & 0.103 & $1.08(0.99,1.18)$ & 0.068 & $1.06(0.98,1.15)$ & 0.147 \\
\hline \multicolumn{7}{|l|}{ Smoking } \\
\hline Never & Reference & & Reference & & Reference & \\
\hline Former & $1.75(0.57,5.42)$ & 0.330 & $1.53(0.32,7.30)$ & 0.593 & $1.73(0.42,7.10)$ & 0.443 \\
\hline Current & $1.87(0.56,6.20)$ & 0.306 & $1.82(0.33,10.13)$ & 0.492 & $0.71(0.10,4.88)$ & 0.724 \\
\hline \multicolumn{7}{|l|}{ Endodontic therapy } \\
\hline No & Reference & & Reference & & Reference & \\
\hline Yes & $7.19(2.37,21.89)$ & $<0.001$ & $8.55(2.43,30.14)$ & $<0.001$ & $8.03(2.38,27.06)$ & $<0.001$ \\
\hline \multicolumn{7}{|l|}{ Vertical furcation } \\
\hline A & Reference & & Reference & & & \\
\hline B-C & $10.30(2.88,36.90)$ & $<0.001$ & $9.83(1.93,50.11)$ & 0.006 & & \\
\hline \multicolumn{7}{|c|}{ Furcation involvement } \\
\hline No & Reference & & & & Reference & \\
\hline Yes & $6.79(2.53,18.25)$ & $<0.001$ & - & & $5.26(1.46,19.03)$ & 0.012 \\
\hline \multicolumn{7}{|l|}{ Intrabony defect } \\
\hline No & Reference & & & & & \\
\hline Yes & $3.53(0.98,12.73)$ & 0.053 & - & & - & \\
\hline Average CAL, mm & $1.41(1.15,1.71)$ & $<0.001$ & - & & - & \\
\hline$\%$ bone loss & $1.09(1.05,1.14)$ & $<0.001$ & - & & - & \\
\hline
\end{tabular}


Table 6. Results of multilevel linear regression for association with furcation involvement at baseline and changes in CAL, \% mesial bone loss and \% distal bone loss

\begin{tabular}{|c|c|c|c|c|c|c|}
\hline & \multicolumn{2}{|l|}{ CAL } & \multicolumn{2}{|c|}{$\%$ mesial bone loss } & \multicolumn{2}{|c|}{$\%$ distal bone loss } \\
\hline & $\begin{array}{l}\text { Coefficients } \\
(95 \% \mathrm{CI})\end{array}$ & $\begin{array}{c}\mathrm{P} \\
\text { value }\end{array}$ & $\begin{array}{l}\text { Coefficients } \\
(95 \% \mathrm{CI})\end{array}$ & $P$ value & $\begin{array}{l}\text { Coefficients } \\
(95 \% \mathrm{CI})\end{array}$ & $P$ value \\
\hline Intercept & $\begin{array}{c}5.39(5.19 \\
5.60)\end{array}$ & $<0.001$ & $0.25(0.23,0.27)$ & $<0.001$ & $0.27(0.25,0.29)$ & $<0.001$ \\
\hline \multicolumn{7}{|l|}{ Time } \\
\hline Time2(APT) & $\begin{array}{c}-0.99(-1.16,- \\
0.82)\end{array}$ & $<0.001$ & $\begin{array}{c}-0.02(-0.04, \\
0.002)\end{array}$ & 0.071 & $\begin{array}{c}-0.01(-0.03, \\
0.01)\end{array}$ & \\
\hline Time3(SPT) & $\begin{array}{c}-1.06(-1.25,- \\
0.87)\end{array}$ & $<0.001$ & $\begin{array}{c}-0.03(-0.06,- \\
0.01)\end{array}$ & 0.011 & $\begin{array}{c}-0.02(-0.05 \\
0.00)\end{array}$ & 0.049 \\
\hline \multicolumn{7}{|c|}{ Furcation involvement } \\
\hline Degree I & $\begin{array}{c}1.06(0.69 \\
1.42)\end{array}$ & $<0.001$ & $0.05(0.02,0.09)$ & 0.004 & $0.06(0.02,0.09)$ & 0.001 \\
\hline Degree II & $\begin{array}{c}2.61(2.13, \\
3.09)\end{array}$ & $<0.001$ & $0.11(0.07,0.16)$ & $<0.001$ & $0.12(0.07,0.16)$ & $<0.001$ \\
\hline Degree III & $\begin{array}{l}4.74(4.15 \\
5.32)\end{array}$ & $<0.001$ & $0.23(0.18,0.29)$ & $<0.001$ & $0.26(0.21,0.31)$ & $<0.001$ \\
\hline \multicolumn{7}{|c|}{$\begin{array}{l}\text { Time * Furcation } \\
\text { involvement }\end{array}$} \\
\hline \multicolumn{7}{|l|}{ Time2(APT) } \\
\hline Degree I & $\begin{array}{c}-0.25(-0.54 \\
0.05)\end{array}$ & 0.104 & $\begin{array}{c}-0.02(-0.05 \\
0.02)\end{array}$ & 0.383 & $0.01(-0.02,0.05)$ & 0.548 \\
\hline Degree II & $\begin{array}{c}-0.64(-1.03,- \\
0.24)\end{array}$ & 0.002 & $\begin{array}{c}-0.03(-0.07 \\
0.01)\end{array}$ & 0.192 & $0.00(-0.05,0.04)$ & 0.856 \\
\hline Degree III & $\begin{array}{c}-1.41(-1.94,- \\
0.89)\end{array}$ & $<0.001$ & $\begin{array}{c}0.05(-0.002 \\
0.10)\end{array}$ & 0.062 & $\begin{array}{c}-0.01(-0.06, \\
0.04)\end{array}$ & 0.759 \\
\hline \multicolumn{7}{|l|}{ Time3(SPT) } \\
\hline Degree I & $\begin{array}{c}-0.27(-0.62 \\
0.08)\end{array}$ & 0.126 & $0.01(-0.03,0.05)$ & 0.654 & $0.03(-0.01,0.07)$ & 0.136 \\
\hline Degree II & $\begin{array}{c}-0.63(-1.09,- \\
0.17)\end{array}$ & 0.007 & $0.03(-0.02,0.08)$ & 0.312 & $0.00(-0.04,0.05)$ & 0.838 \\
\hline Degree III & $\begin{array}{c}-0.93(-1.55,- \\
0.31)\end{array}$ & 0.003 & $0.07(0.01,0.13)$ & 0.034 & $\begin{array}{c}-0.02(-0.08, \\
0.04)\end{array}$ & 0.532 \\
\hline
\end{tabular}

\section{FIGURE LEGEND}

Figure 1. Treatment breakdown according to furcation diagnosis 